Supporting Information

\title{
Zinc Oxide and Metal Halide Perovskite Nanostructures Having Tunable Morphologies Grown by Nanosecond Laser Ablation for Light-Emitting Devices
}

Nazrin Abdullayeva', Cigdem Tuc Altafl, Alihan Kumtepe', Nazmi Yilmaz², Ozlem Coskun', Mehmet Sankirl, 3,*, Hamza Kurt1,2,3, Cem Celebi", Alper Yanilmaz", Nurdan Demirci Sankirı, 3,*

'Micro and Nanotechnology Graduate Program, TOBB University of Economics and Technology, Sogutozu Caddesi No 43 Sogutozu 06560 Ankara, Turkey

2Department of Electrical and Electronics Engineering, TOBB University of Economics and Technology, Sogutozu Caddesi No 43 Sogutozu 06560 Ankara, Turkey

${ }^{3}$ Department of Materials Science and Nanotechnology Engineering, TOBB University of Economics and Technology, Sogutozu Caddesi No 43 Sogutozu 06560 Ankara, Turkey

${ }^{4}$ Quantum Device Laboratory, Department of Physics, İzmir Institute of Technology, Gulbahce Campus, Urla 35430 Izmir, Turkey

*Correspondending author email: $\underline{\text { msankir@etu.edu.tr ; nsankir@etu.edu.tr }}$ 


\section{Laser Parameters and Details of Calculations}

Fluence energy of our system has been calculated via using the following equations.

$$
\begin{aligned}
& \text { Fluence Energy } \left.=\text { Pulse Energy }(J) / \text { Area of the spot size }\left(\mathrm{cm}^{2}\right) \quad \text { (Equation } \mathrm{S} 1\right) \\
& \text { \& } \\
& \text { Pulse Energy }=\text { Average Power }(W) / \text { Frequency }(\mathrm{Hz}) \quad \text { (Equation S2) }
\end{aligned}
$$

Table S1 summarizes some properties of the laser system used in this work.

Table S1. Basic Laser Parameters of Nanosecond Laser

\begin{tabular}{|c|c|}
\hline Laser Source & Nd-YAG (Y3AL5012) \\
\hline Laser Pulse Type & Nanosecond(ns) \\
\hline Wavelength & $1064 \mathrm{~nm}$ \\
\hline Range of Average Power & $0.008-25 \mathrm{Watt}$ \\
\hline Range of Frequency & $70-200 \mathrm{kHz}$ \\
\hline Focal Length & $160 \mathrm{~mm}$ \\
\hline Depth of Focus & $181 \mathrm{~mm}$ \\
\hline Diameter of Light from Flat(D) & $7.5 \mathrm{~mm}$ \\
\hline Correction Factor & $1.4 \mathrm{~m}^{2}$ \\
\hline Pulse Duration & $160 \mathrm{~ns}$ \\
\hline
\end{tabular}



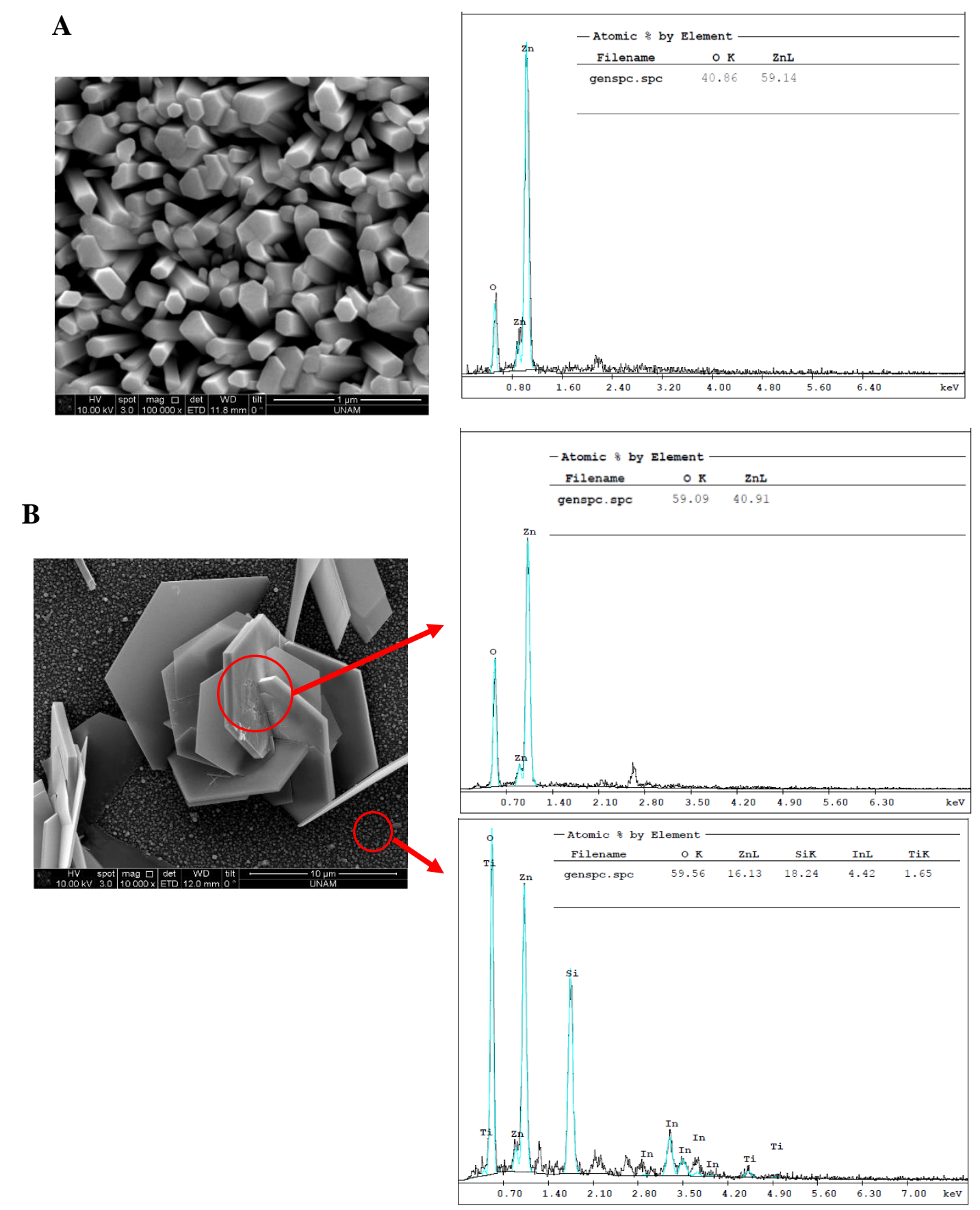

Figure S1. EDAX data indicating elemental ratios obtained from (A) NR and (B) NF thin films. 

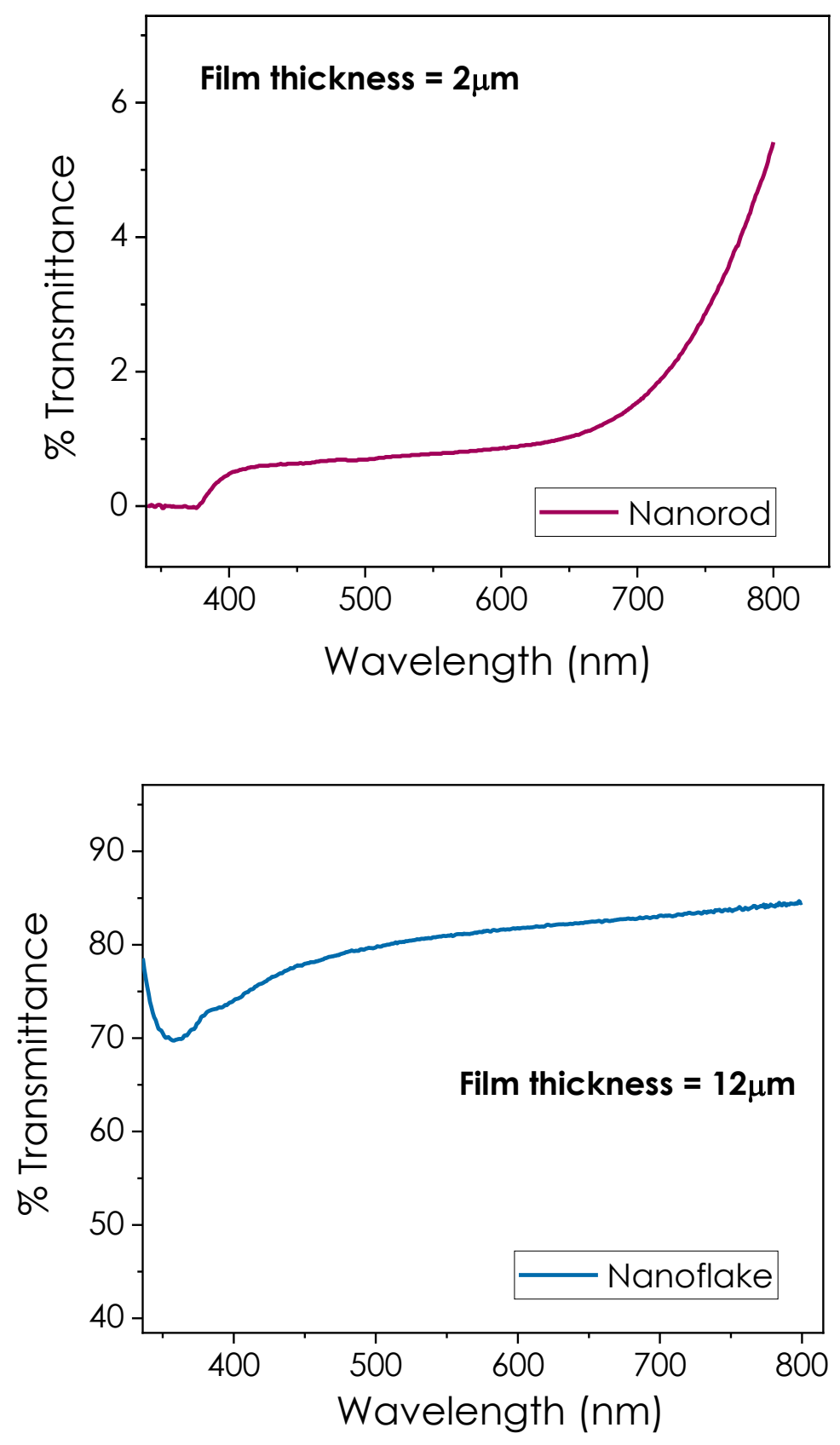

Figure S2. Transmittance values and film thicknesses used for Tauc plot evaluation and $\mathrm{E}_{\mathrm{g}}$ calculations. 
A

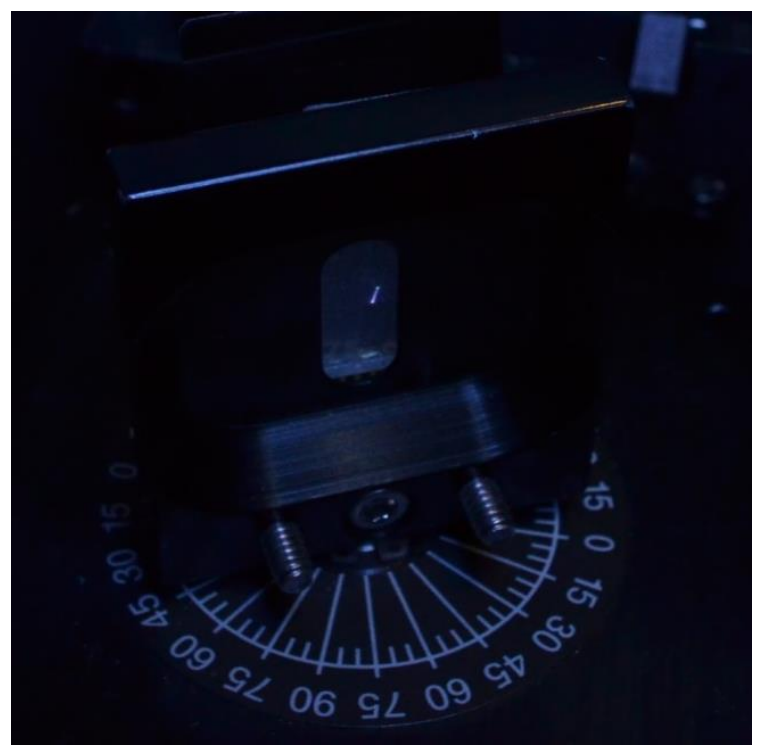

B

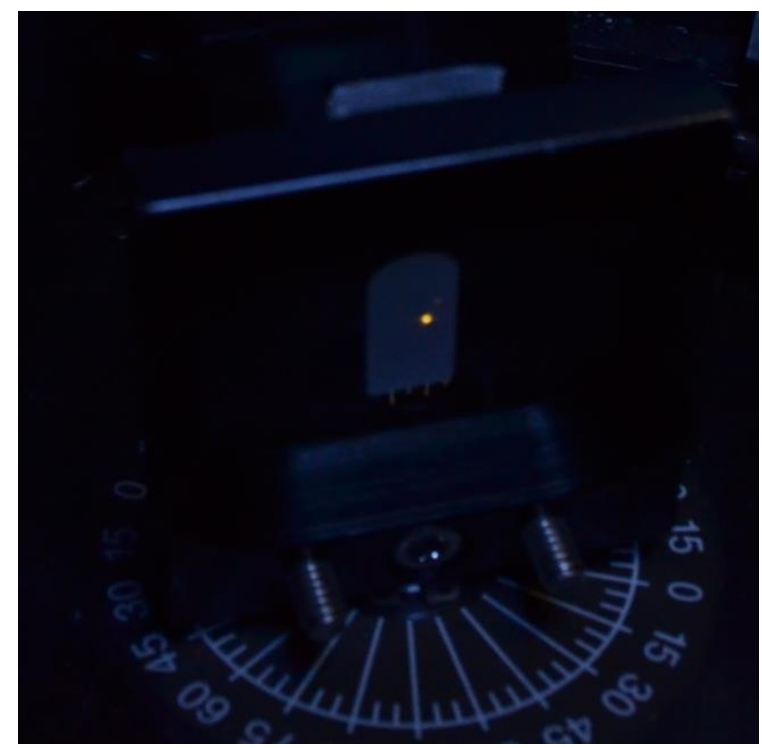

Figure S3. Emissions obtained under 325nm He:Cd laser excitation from (A) Nanoflake and (B) Nanorod samples. 

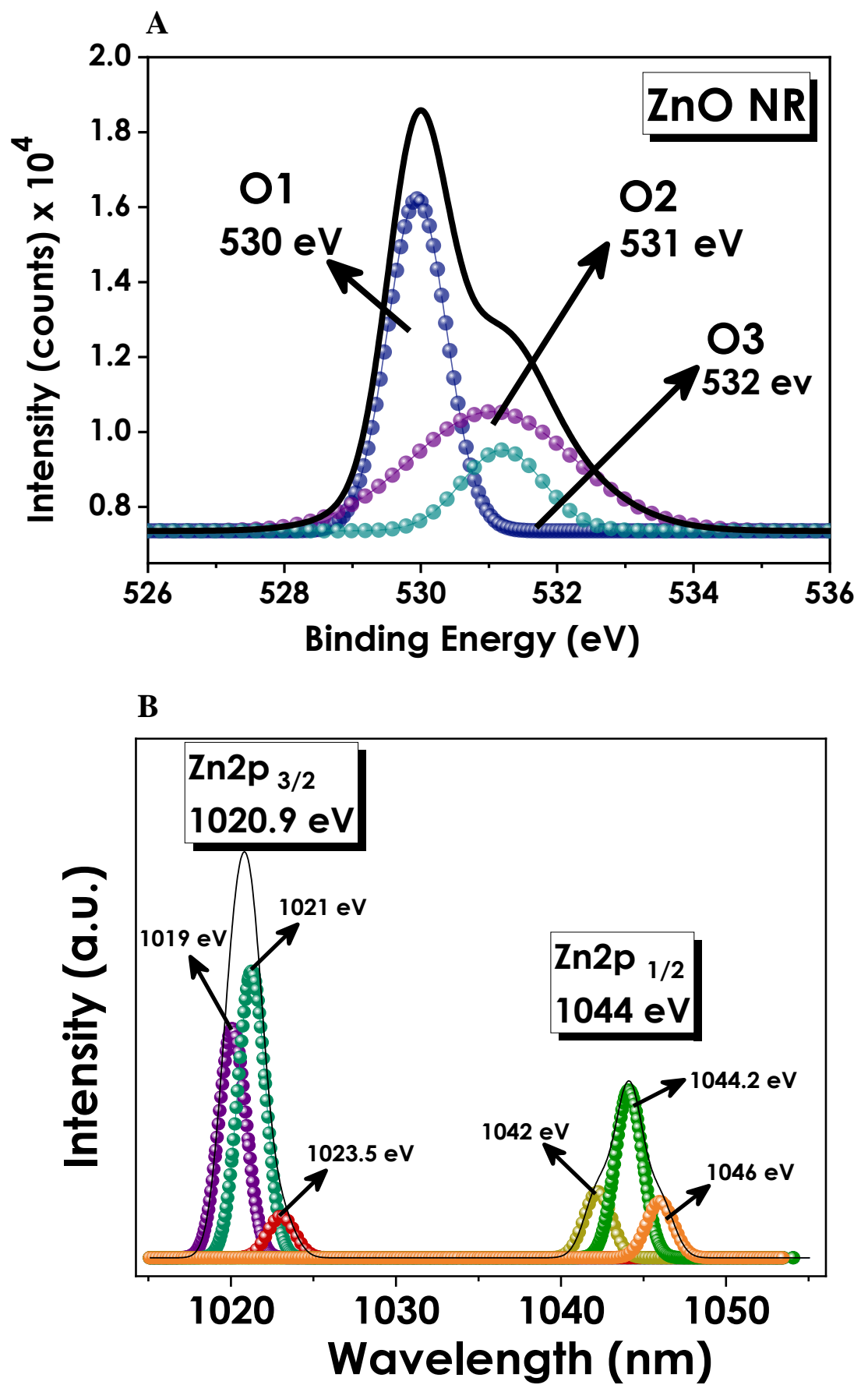

Figure S4. XPS analyses obtained from ZnO NRs showing the individual (A) O1s and (B) Zn2p deconvolutions.. 
Table S2. Decay periods obtained from TrPL spectra of NR and NF with corresponding collection wavelengths

\begin{tabular}{|c|c|c|c|c|}
\hline ZnO & $\begin{array}{c}\text { Acollection } \\
(\mathbf{n m})\end{array}$ & T1 (ns) & T2 (ns) & Tavg (ns) \\
\hline NR & 650 & 4.52 & 53.33 & 28.92 \\
\hline NF & 550 & 1.80 & N.A. & 1.80 \\
\hline NF & 750 & 1.51 & 11.90 & 6.71 \\
\hline
\end{tabular}



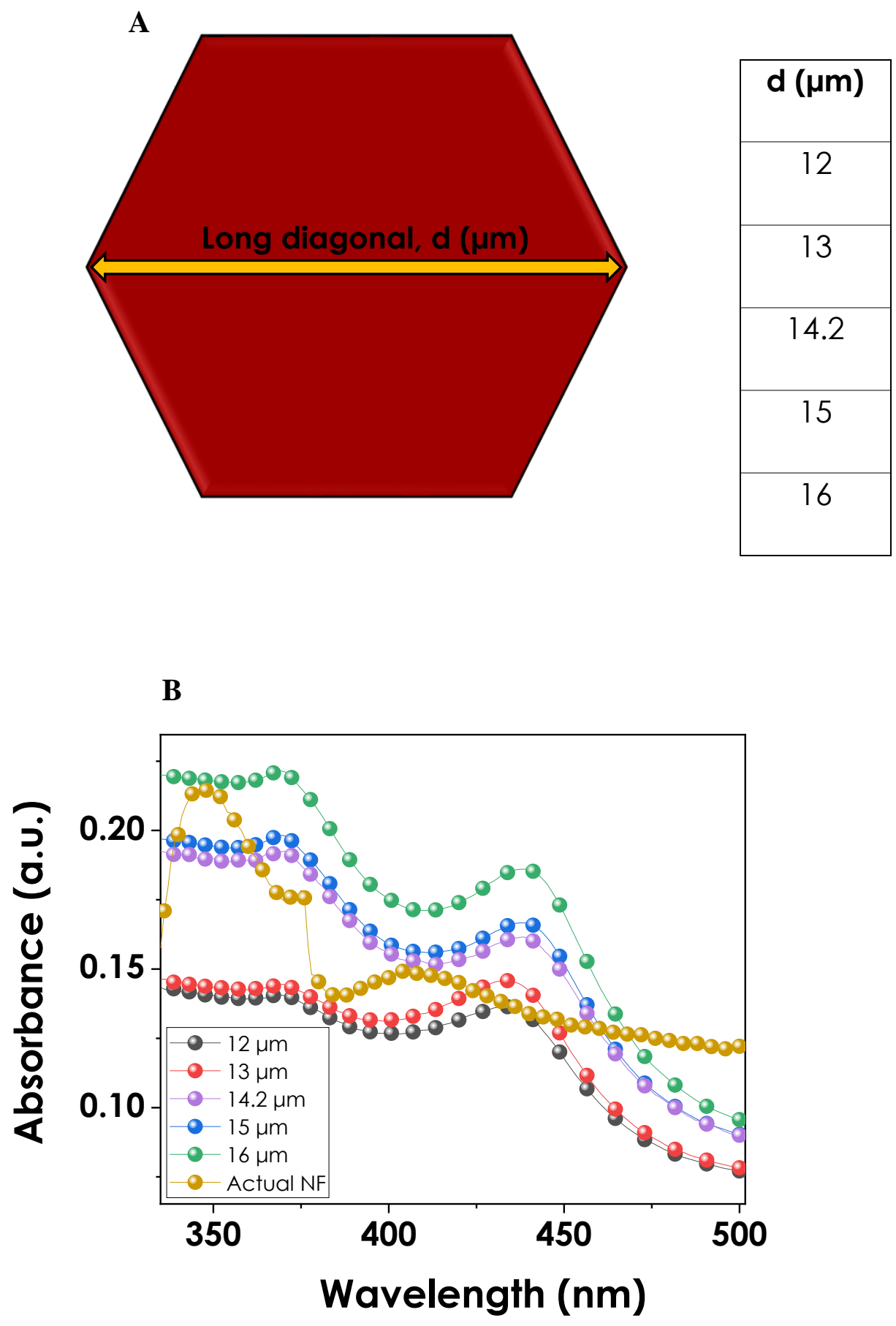

Figure S5. (A) Hexagonally shaped NF with specified long diagonal (d) used in FDTD simulations. (B) Absorbance spectra of NFs at differed $d$ values compared with the experimental results 

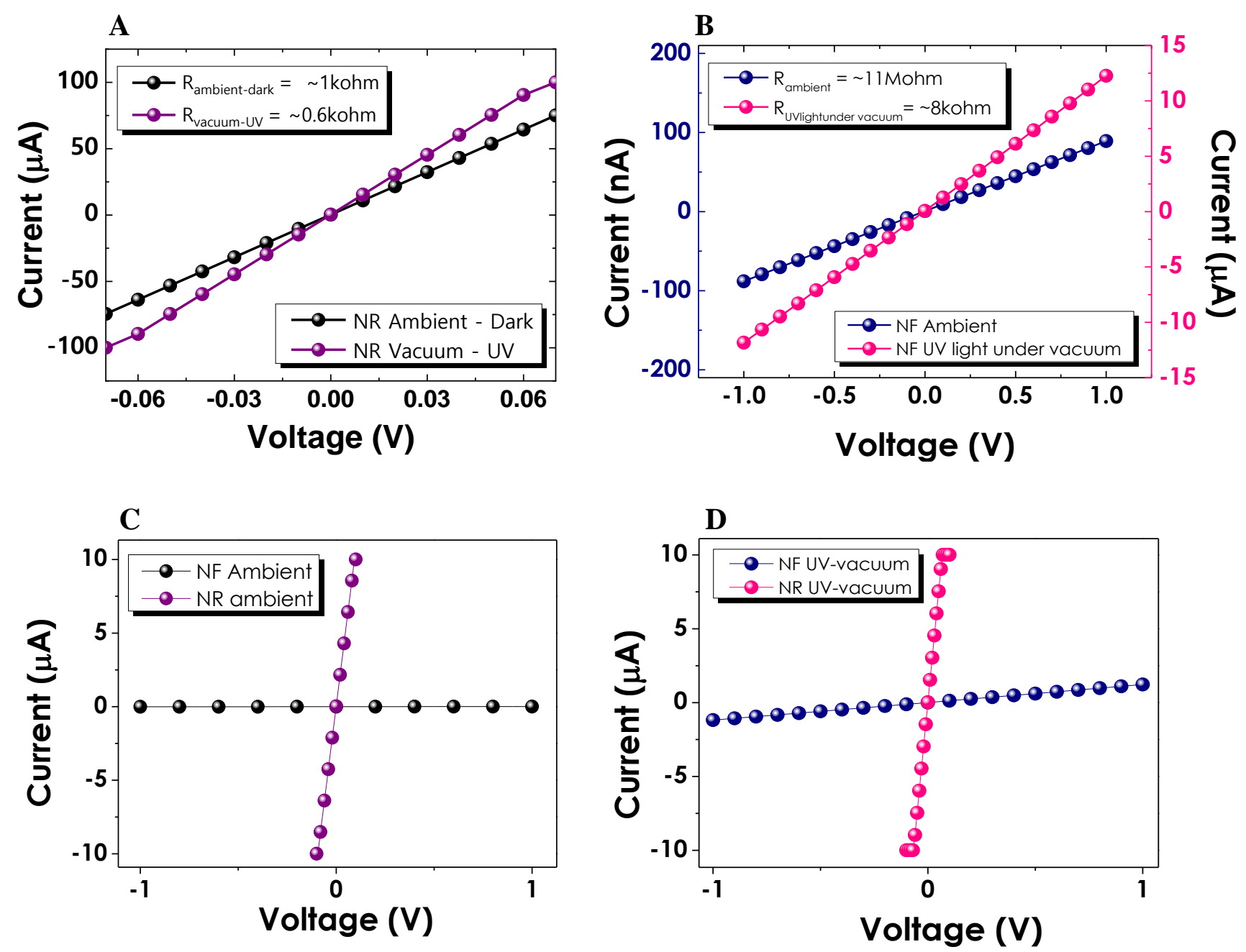

Figure S6. I-V curves obtained under condition 1 (C1 - UV illuminated vacuum) and condition 2 (C2 - dark ambient air) for; (A) Nanorod, (B) Nanoflake samples and individual comparison of nanostructures for (C) $\mathrm{C} 1$ and (D) $\mathrm{C} 2$ conditions. 
A
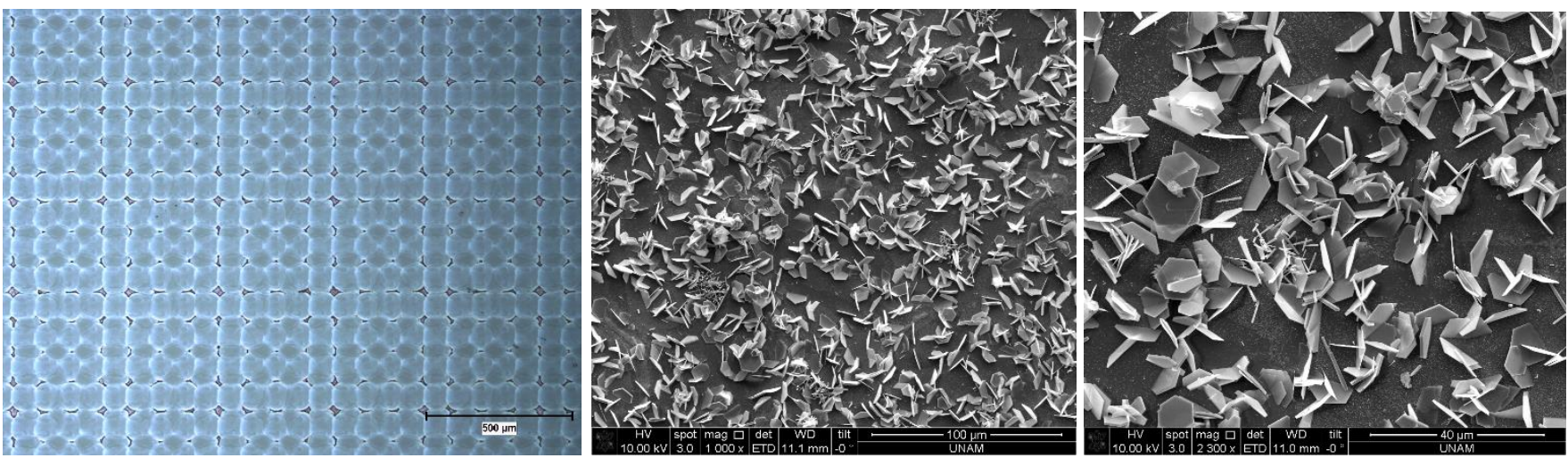

B
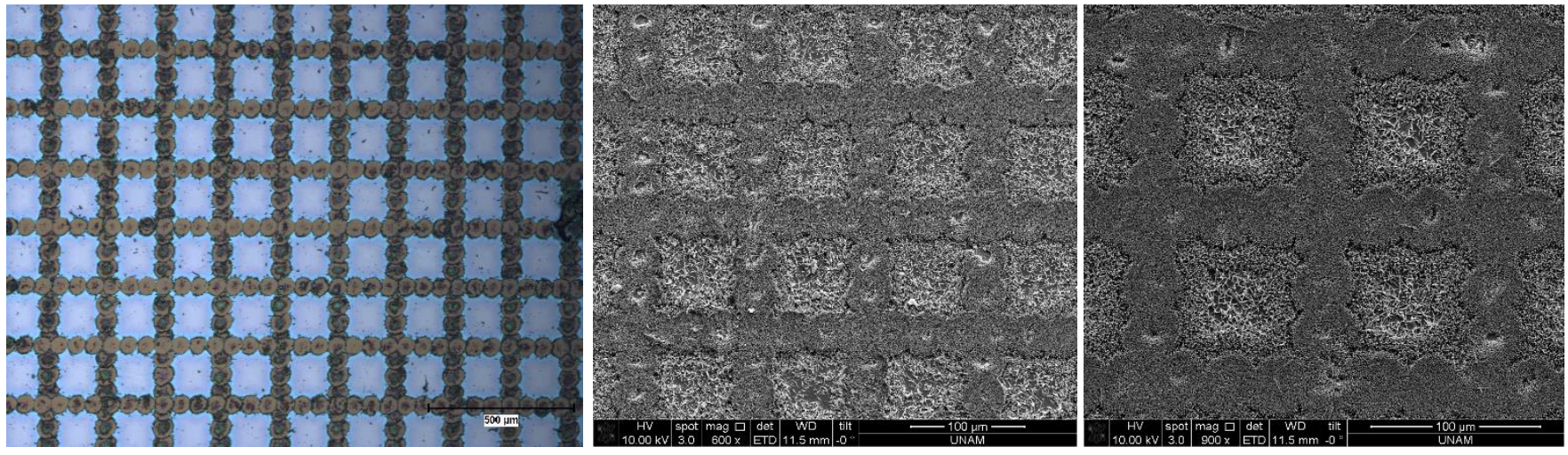

Figure S7. OM images of $0.1 \times 0.1 \mathrm{~cm}^{2}$ bare laser patterned substrates with their corresponding CBD grown SEM images under (A) 25W, $5500 \mathrm{~mm} / \mathrm{s}, 70 \mathrm{kHz}$ and (B) 7W, $4500 \mathrm{~mm} / \mathrm{s}, 100$ $\mathrm{kHz}$ application. 


\section{Necessary COMSOL Multiphysics equations and parameters}

$$
\begin{gathered}
{\left[c_{p}+L_{m} \delta\left(T-T_{m}\right)+L_{v}\left(T-T_{v}\right) \rho\right](\partial T(x, y, t) / \partial t)+\nabla \cdot[-k \nabla T]=Q \quad \text { (Equation S3) }} \\
\delta\left(T-T_{m, v} \Delta\right)=\frac{1}{\sqrt{2 \pi \Delta}} \exp \left[\frac{-\left(T-T_{m, v}\right)^{2}}{2 \Delta^{2}}\right] \quad \text { (Equation S4) } \\
Q(r, z, t)=\frac{2 \varphi_{0} \alpha(\lambda)}{\sqrt{\frac{\pi}{\ln 2 \tau_{p}}}}(1-R(\lambda)) \exp \left[\frac{2 r^{2}}{\omega_{0}^{2}}-(4 \ln 2)\left(t / \tau_{p}\right)^{2}-(\alpha(\lambda) z)\right] \quad \text { (Equation S5) }
\end{gathered}
$$

\begin{tabular}{|c|c|c|c|}
\hline Parameter & Significance & Unit & \multirow{14}{*}{$R^{\prime}(\lambda)=\left[\frac{\left(n_{\text {Ti-ZnO-ITO }}-n_{\text {glass }}\right)}{\left(n_{\text {Ti-ZnO-ITO }}-n_{\text {glass }}\right)}\right]^{2}$} \\
\hline$k$ & Thermal conductivity & $\mathrm{W} \cdot \mathrm{m}^{-1} \cdot \mathrm{K}^{-1}$ & \\
\hline$\rho$ & Material density & Kg.m ${ }^{-3}$ & \\
\hline $\mathrm{T}_{\mathrm{m}}$ & Melting temperature & $\bar{K}$ & \\
\hline $\mathrm{c}_{\mathrm{p}}$ & Heat capacity at constant $\mathrm{P}$ & $\mathrm{J} \cdot \mathrm{kg}^{-1} \cdot \mathrm{K}^{-1}$ & \\
\hline $\mathrm{L}_{\mathrm{m}}$ and $\mathrm{L}_{\mathrm{v}}$ & $\begin{array}{l}\text { Latent heat of fusion and } \\
\text { vaporisation }\end{array}$ & ${\mathrm{J} . \mathrm{kg}^{-1}}^{-1}$ & \\
\hline Q & Laser pulse energy source & W.m-3 & \\
\hline$\varphi_{0}$ & Peak fluence & J. $\mathrm{m}^{-2}$ & \\
\hline $\mathrm{R}(\lambda)$ & Film reflectivity & - & \\
\hline$\tau_{p}$ & $\begin{array}{l}\text { Full width half maximum of } \\
\text { pulse duration }\end{array}$ & $\mathrm{s}$ & \\
\hline $\mathrm{r}$ & Radial coordinate & $\mathrm{m}$ & \\
\hline$\omega_{0}$ & $1 / \mathrm{e}^{2}$ of spot radius & $\mathrm{m}$ & \\
\hline$a(\lambda)$ & $\begin{array}{l}\text { Material absorption } \\
\text { coefficient }\end{array}$ & $\mathrm{m}^{-1}$ & \\
\hline $\mathrm{z}$ & Depth component & $\mathrm{m}$ & \\
\hline
\end{tabular}

Table S3. Parameters used for COMSOL Multiphysics simulation with their significances and units

(Equation S6) 
A

$200 \mu \mathrm{m}$

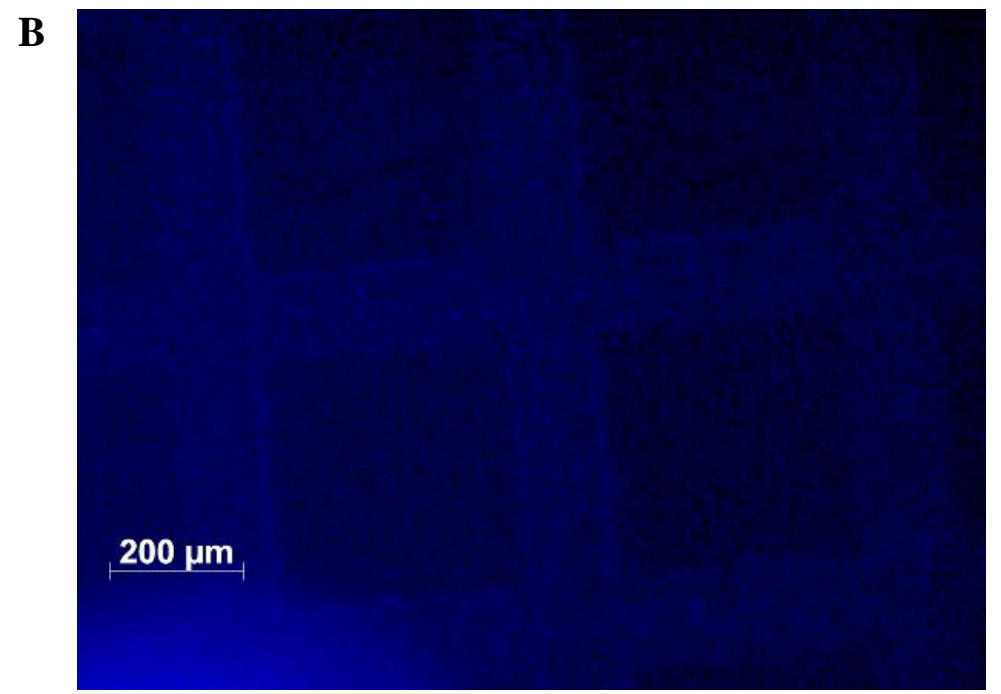

Figure S8. Fluorescence microscopy images of laser patterned and CBD grown samples under UV illumination (365nm) for (A) $3.72{\mathrm{~J} . \mathrm{cm}^{-2}}^{-2}$ patterned samples and (B) $28.43 \mathrm{~J} . \mathrm{cm}^{-2}$ patterned samples. 


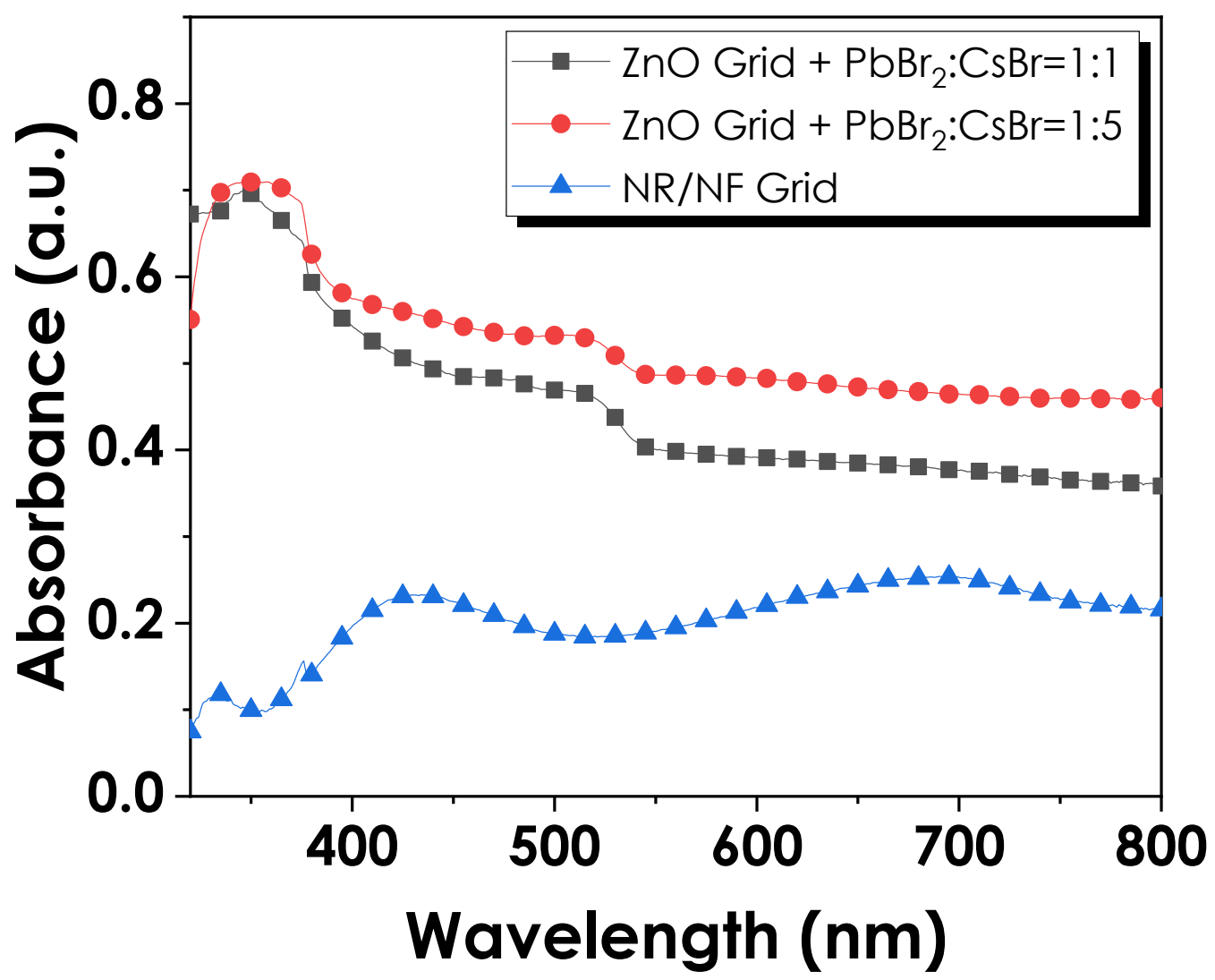

Figure S9. Absorbance comparison between pristine and perovskite deposited $\mathrm{ZnO}$ grid samples within UV/Vis wavelength range 


\section{Crystal Size Calculation via Scherrer's Equation}
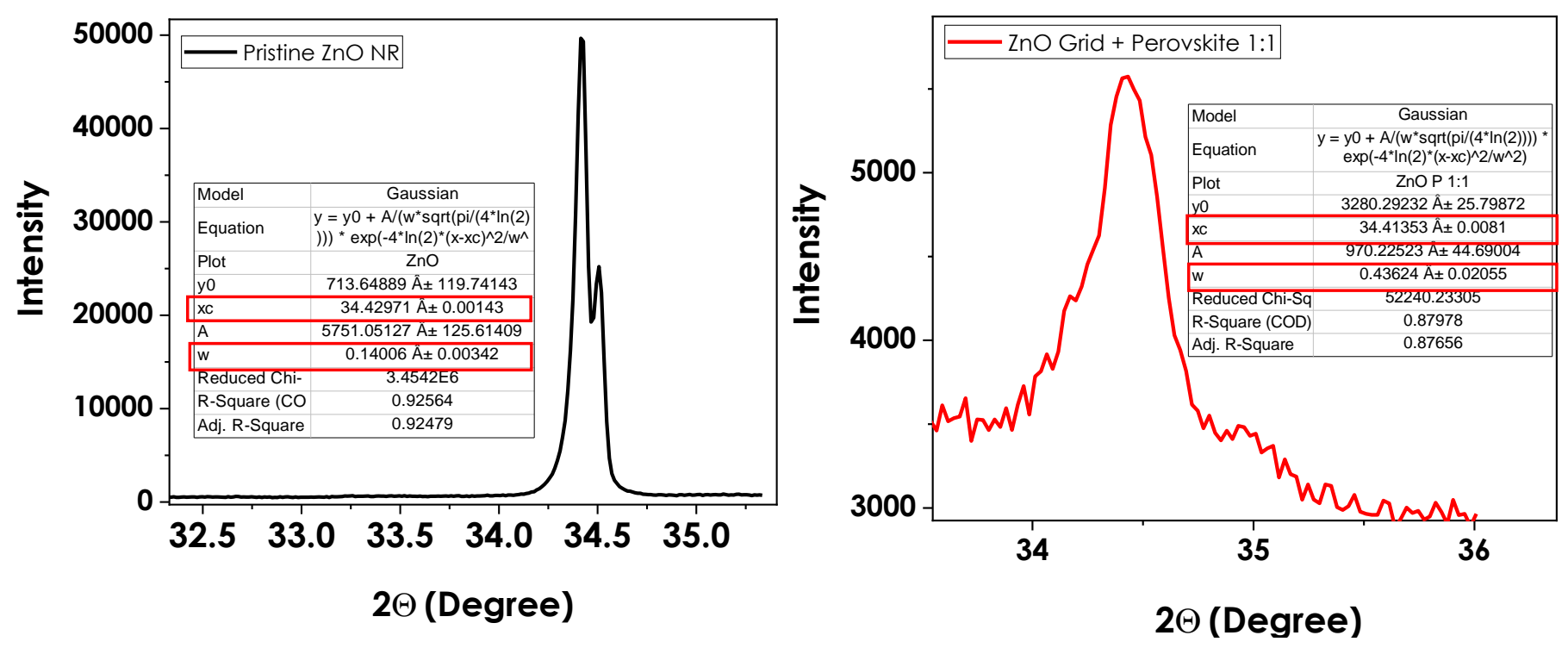

Figure S10. XRD crystal size evaluation plots for pristine and perovskite deposited $\mathrm{ZnO}$

$$
\begin{aligned}
& D=\frac{k \lambda}{\beta \cos \theta} \\
& k=0.90 \text { Scherrer constant } \\
& \lambda=0.154 \mathrm{~nm} \\
& \mathrm{Xc}=\text { Peak position }(2 \theta) \\
& w=\beta=F W H M \text { (radians) }
\end{aligned}
$$

\begin{tabular}{|c|c|c|}
\hline \multicolumn{3}{|c|}{$\mathrm{ZnO}$} \\
\hline Peak position $2 \Theta$ & FWHM & Crystal Size \\
\hline 34.43 & 0.14 & 58.97 \\
\hline
\end{tabular}

\begin{tabular}{|c|r|r|}
\hline \multicolumn{3}{|c|}{$\mathrm{ZnO}+$ Perovskite } \\
\hline Peak position $2 \Theta$ & FWHM & Crystal Size \\
\hline 34.41 & 0.43 & 19.45 \\
\hline
\end{tabular}




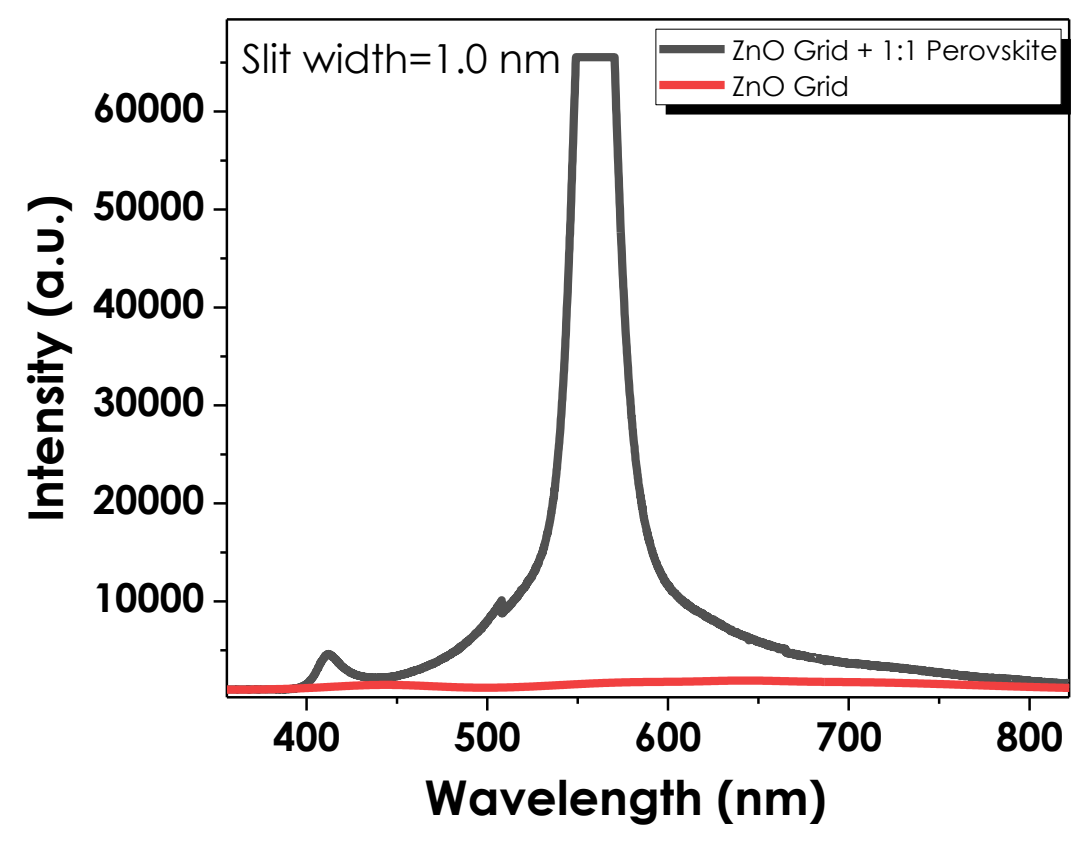

Figure S11. Comparison between PL intensities of pristine and 1:1 perovskite deposited $\mathrm{ZnO}$ samples under the same slit number. 